\title{
Remodeling after acute myocardial infarction: mapping ventricular dilatation using three dimensional CMR image registration
}

\author{
Declan P O'Regan ${ }^{1 *}$, Wenzhe Shi ${ }^{2}$, Ben Ariff ${ }^{3}$, A John Baksi ${ }^{4}$, Giuliana Durighel ${ }^{1}$, Daniel Rueckert ${ }^{2}$
} and Stuart A Cook'

\begin{abstract}
Background: Progressive heart failure due to remodeling is a major cause of morbidity and mortality following myocardial infarction. Conventional clinical imaging measures global volume changes, and currently there is no means of assessing regional myocardial dilatation in relation to ischemic burden. Here we use 3D co-registration of Cardiovascular Magnetic Resonance (CMR) images to assess the long-term effects of ischemia-reperfusion injury on left ventricular structure after acute ST-elevation myocardial infarction (STEMI).

Methods: Forty six patients (age range 33-77 years) underwent CMR imaging within 7 days following primary percutaneous coronary intervention (PPCI) for acute STEMI with follow-up at one year. Functional cine imaging and Late Gadolinium Enhancement (LGE) were segmented and co-registered. Local left ventricular wall dilatation was assessed by using intensity-based similarities to track the structural changes in the heart between baseline and follow-up. Results are expressed as means, standard errors and 95\% confidence interval (Cl) of the difference.

Results: Local left ventricular remodeling within infarcted myocardium was greater than in non-infarcted myocardium ( $1.6 \% \pm 1.0$ vs $0.3 \% \pm 0.9,95 \% \mathrm{Cl}:-2.4 \%--0.2 \%, \mathrm{P}=0.02)$. One-way ANOVA revealed that transmural infarct thickness had a significant effect on the degree of local remodeling at one year $(P<0.0001)$ with greatest wall dilatation observed when infarct transmurality exceeded 50\%. Infarct remodeling was more severe when microvascular obstruction (MVO) was present $(3.8 \% \pm 1.3 \mathrm{vs}-1.6 \% \pm 1.4,95 \% \mathrm{Cl}:-9.1 \%--1.5 \%, \mathrm{P}=0.007)$ and when end-diastolic volume had increased by $>20 \%(4.8 \% \pm 1.4$ vs $-0.15 \% \pm 1.2,95 \% \mathrm{Cl}:-8.9 \%--0.9 \%, \mathrm{P}=0.017)$.

Conclusions: The severity of ischemic injury has a significant effect on local ventricular wall remodeling with only modest dilatation observed within non-ischemic myocardium. Limitation of chronic remodeling may therefore depend on therapies directed at modulating ischemia-reperfusion injury. CMR co-registration has potential for assessing dynamic changes in ventricular structure in relation to therapeutic interventions.
\end{abstract}

Keywords: Cardiovascular magnetic resonance, Acute myocardial infarction, Image analysis

\section{Background}

Since the advent of primary percutaneous coronary intervention (PPCI) to treat acute myocardial infarction immediate survival has improved but at the expense of a rising incidence of progressive heart failure [1]. Ischemiareperfusion injury leads to a sequence of events that result in predictable changes to the structure and function

\footnotetext{
* Correspondence: declan.oregan@imperial.ac.uk

'Robert Steiner MRI Unit, MRC Clinical Sciences Centre, Imperial College London, Hammersmith Hospital Campus, Du Cane Road, London W12 ONN, UK

Full list of author information is available at the end of the article
}

of the left ventricle (LV) that may eventually cause congestive heart disease [2]. Our understanding of remodeling is largely based on animal models which show that within the first few days after coronary occlusion there is slippage and stretching of myocytes in the infarcted zone [3]. Late remodeling also involves changes to the nonischemic myocardium as it adapts to the extra load placed on it by dilating $[4,5]$. Current methods of evaluating remodeling rely on measuring global changes in left ventricular volume, however this does not reveal where myocardial enlargement and dilatation occur within the ventricle or how remodeling is influenced by the severity 
of local ischemic injury. Determining the pattern of left ventricular remodeling in patients and the contribution made by infarcted and remote myocardium to chamber dilatation has importance for evaluating interventions aimed at preventing heart failure [6].

In this paper we apply techniques previously developed in neuroscience research to align and co-register Cardiovascular Magnetic Resonance (CMR) images of the heart to map the effects of remodeling in three dimensions [7]. This framework enables highly consistent comparisons to be made between different CMR sequences obtained at various time-points [8]. By tracking how each point on the myocardial surface changes relative to neighboring points we can build a three dimensional model of how the LV remodels over time. Co-registering structural imaging with late-enhancement sequences also allows the extent of local remodeling to be compared to the degree of ischemic injury present. However, developing these techniques in the heart is challenging as it requires a consistent and accurate approach to co-registering and transforming the cardiac MR images acquired in a longitudinal study.

The aim of this study was to use $3 \mathrm{D}$ co-registration techniques to determine how the LV remodels after reperfused acute ST-elevation myocardial infarction (STEMI) and the relationship to ischemic injury.

\section{Methods}

\section{Participants}

The study was approved by the Hospital's research ethics committee and all patients gave written informed consent. To be included in the study each patient had to have been admitted within 24 hours of the onset of chest pain with an ECG diagnosis of acute STEMI and angiographically proven partial or complete coronary occlusion of the infarct-related artery. Exclusion criteria were contraindications to CMR, previous $\mathrm{MI}$ or heart failure, clinical instability, significant arrhythmias, pregnancy or lactation. In total $\mathrm{MR}$ images from 46 patients were analyzed (44 male, 2 female; age range 33 to 77 years; mean age 55 years). A baseline CMR was performed within 1 week of PPCI and the follow-up study at one year.

\section{Coronary intervention}

Coronary catheterization was used to identify and treat the infarct-related artery and all patients received either a bare-metal or drug-eluting stent. Standard medical treatment was provided. Flow in the infarct-related artery was graded using the Thrombolysis in Myocardial Infarction trial (TIMI) criteria prior to and after intervention [9].

\section{CMR protocol}

The CMR studies were performed on a $1.5 \mathrm{~T}$ Philips Achieva system (Best, Netherlands) using a 32-channel coil. Myocardial edema was imaged with a navigatorgated black blood T2-weighted turbo spin echo sequence with spectrally selective inversion recovery (SPIR) fat suppression using the following parameters - field of view 350 × $280 \mathrm{~mm}$; matrix, 256 x 123; repetition time msec/echo time msec, $2 \mathrm{R}-\mathrm{R}$ intervals/100; in-plane resolution, $1.4 \times 2.3 \mathrm{~mm}$; section thickness, $8 \mathrm{~mm}$. An intravenous bolus of Gadobutrol $1.0 \mathrm{mmol} / \mathrm{ml}$ (Gadovist; Bayer Schering Pharma, Berlin, Germany) was administered at a dose of $0.15 \mathrm{mmol} / \mathrm{kg}$. Early enhancement imaging was performed to assess microvascular obstruction (MVO) with a single breath hold 3D inversion recovery sequence within 1 minute of contrast injection using the following parameters - field of view, 350 x $290 \mathrm{~mm}$; matrix $252 \times 135$; repetition time msec/echo time msec, 4.4/1.3; in-plane resolution, $1.4 \times 2.1 \mathrm{~mm}$; section thickness $8 \mathrm{~mm}$. Late Gadolinium Enhancement (LGE) was performed to assess infarct size 10 minutes after contrast injection with a $2 \mathrm{D}$ inversion recovery sequence using the following parameters - field of view, 320 x $320 \mathrm{~mm}$; matrix $200 \times 147$; repetition time msec/echo time msec, 5.5/2.5; in-plane resolution $1.6 \times 2.2 \mathrm{~mm}$; section thickness $8 \mathrm{~mm}$. Balanced-steady state free precession (b-SSFP) images in the left ventricular short axis plane were acquired using the following parameters - field of view 320 × $320 \mathrm{~mm}$; matrix, 160 x 151; repetition time msec/echo time msec, 3.0/1.5; shot duration, $50 \mathrm{~ms}$; in-plane resolution, $2.0 \times 2.2 \mathrm{~mm}$; number of cardiac phases, 30; section thickness, $8 \mathrm{~mm}$.

\section{Image segmentation and registration}

Our approach was to co-register the cine and LGE images onto an atlas template, and then use intensitybased similarities within the heart to track local expansion of the LV in the first year after acute STEMI (Figure 1). The atlas comprised a set of manually segmented end-diastolic images where each voxel is assigned a probability of being blood pool, left ventricular wall, right ventricle blood pool or background structures (Figure 2) [10]. The prior information in the atlas was used in the segmentation of the target images and to correct for differences in alignment between individual subjects and MR acquisitions. The atlas was registered to the target image using affine, local affine and then nonrigid registration [11]. We used an expectation-maximum (EM) technique with spatial information from the probabilistic atlas to segment the LV from surrounding structures [10]. The accuracy of the automatic segmentation was assessed by manually defining the endocardium and epicardium on the same image sets and quantifying the mean surface-to-surface distances between the respective segmentations. The baseline LGE images were then rigidly aligned to the baseline cine short axis stack to co-register structural imaging with the distribution of 


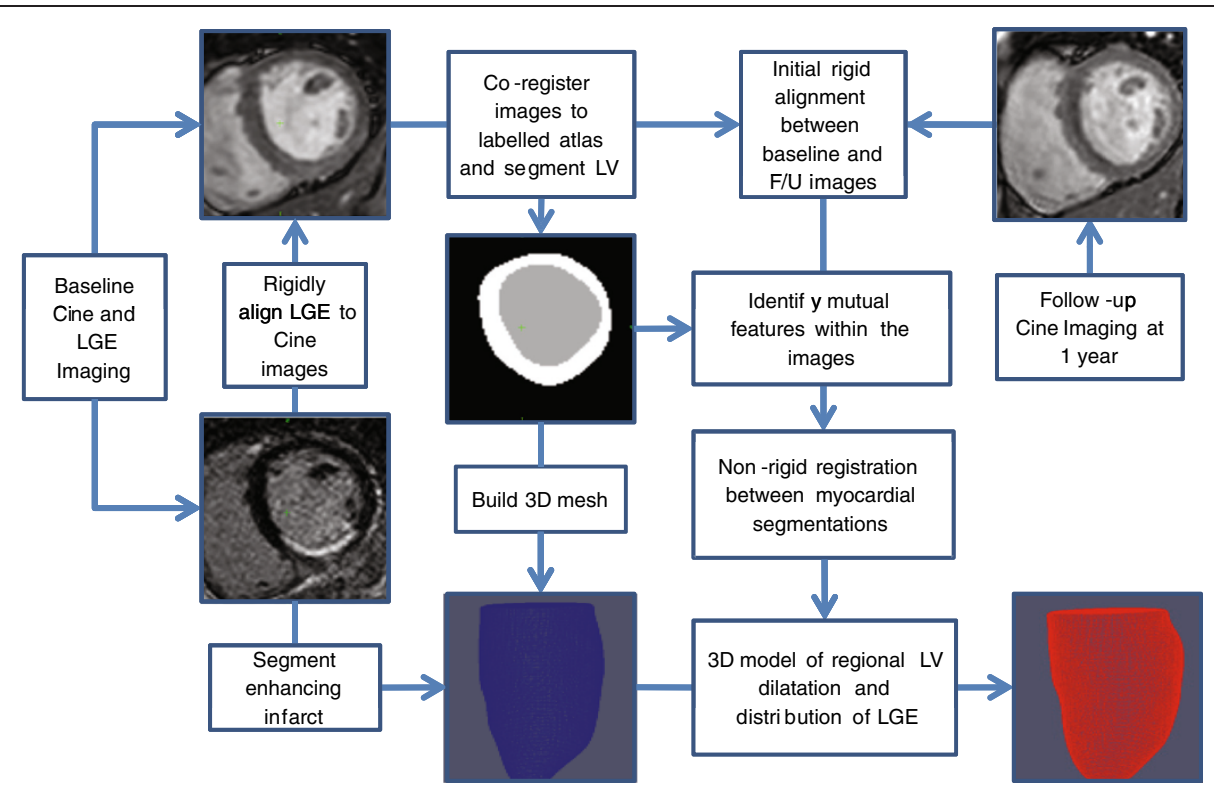

Figure 1 Flow chart showing the steps in image co-registration. The cardiac atlas labels are co-registered to the baseline cine and LGE sequences to segment the myocardium. The infarct itself is then segmented using a Gaussian mixture model. The baseline and follow-up cine images are first rigidly aligned for anatomical consistency. A non-rigid registration is then used to build a map of local ventricular dilatation by tracking the relative position of intrinsic landmarks shared by the images.

infarcted tissue. We used a two-component Gaussian mixture model (GMM) to discriminate between enhancing and non-enhancing voxels on the LGE images [12]. The distribution with a higher mean signal intensity mean was considered as the infarct intensity distribution. The transmural extent of infarction was calculated as the proportion of infarcted myocardium in the radial direction of the left ventricular wall. Wall thickness was calculated along lines joining an epicardial vertex to the nearest endocardial vertex.

A 3D mesh reconstruction of the endocardial and epicardial surfaces was created from the baseline segmentation $[13,14]$. To make longitudinal comparisons the baseline and follow-up reconstructions were first rigidly aligned using intensity information from the whole image to achieve anatomical consistency. A non-rigid registration between the segmentations at each visit was then used to measure how the ventricle deforms over time by tracking the relative position of corresponding points in the myocardium. This approach uses normalized mutual information in the images to identify matching anatomical features and signal intensity patterns to use as landmarks (Figure 3) [11]. As the LV remodels and the myocardium expands the relative separation of corresponding points on the mesh reconstruction increases (Figure 4). Local ventricular remodeling was
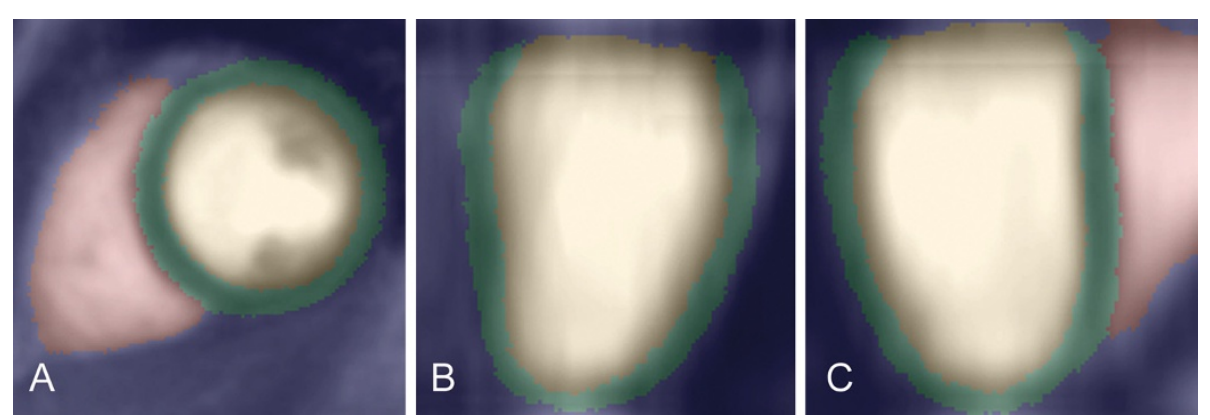

Figure 2 Probabilistic cardiac atlas. A labeled probabilistic atlas was used to provide prior knowledge of the cardiac anatomy during the segmentation process. Multiplanar reconstructions of the atlas are shown where each voxel is assigned a probability of belonging to left ventricular blood pool (yellow), left ventricular wall (green), right ventricle blood pool (red) and background (blue). (A) Left ventricular short axis. (B) Vertical long axis. (C) Horizontal long axis. 

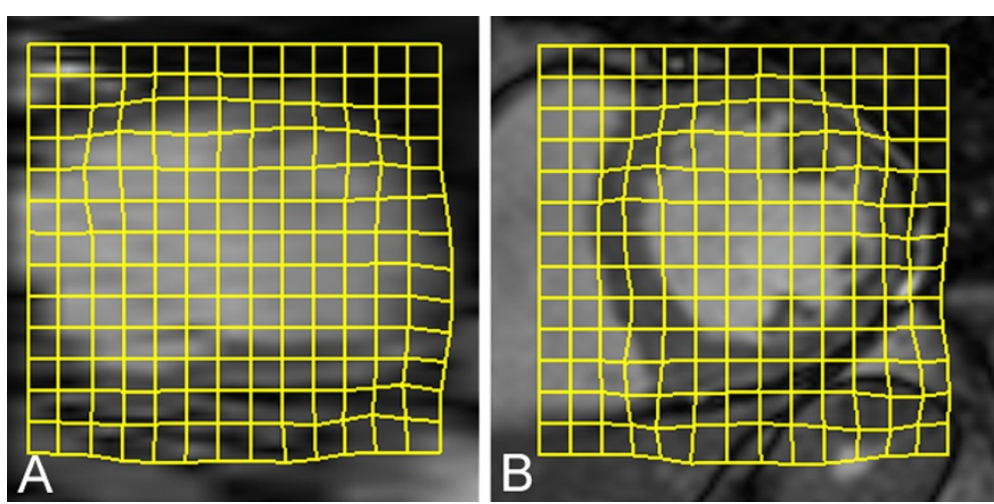

Figure 3 Image transformation between time-points. A non-rigid transformation (yellow grid) between the baseline cine images and those obtained at follow-up is used to assess regional remodeling in three dimensions. Intrinsic features within the image are used to track how each part of the ventricular wall deforms and dilates over time as a consequence of infarction. (A) Long axis. (B) Short axis.

defined as the mean change in separation between each vertex on the mesh and its surrounding neighbors $[5,15]$ and was therefore independent of infarct size.

The presence of MVO was defined as a region of low signal intensity within the infarcted region on early enhancement images [16]. Left ventricular mass, end diastolic volume and end systolic volume were measured using CMRtools (Cardiovascular Imaging Solutions, London, UK). Global left ventricular remodeling was considered to be present if there was at least a $20 \%$ increase in end diastolic volume between baseline and follow-up [17].

\section{Statistical analysis}

Data were analyzed with IBM SPSS statistics (IBM, Chicago, Ill). Remodeling data are presented as mean and standard error of the mean. Comparisons were made using two tailed t-tests and reported as a $\mathrm{P}$ value with the $95 \%$ confidence interval (CI) of the difference of the mean. The effect of infarct thickness on remodeling was evaluated with a one-way analysis of variance (ANOVA). A $P$ value $<0.05$ was considered significant. As this was a preliminary study no correction for multiple tests was made.

\section{Results}

Baseline characteristics for all patients are shown in Table 1. The baseline CMR was performed at a mean of 2.7 days (range $1-7$ days) post-PPCI and the follow-up study at a mean of 54 weeks (range 53 - 57 weeks). All subjects demonstrated an enhancing infarct on the LGE images within the vascular territory of the culprit coronary artery corresponding to high signal on T2-weighted imaging. No patients demonstrated co-existing chronic infarctions or infarcts in multiple coronary territories. Mean infarct size was $13.3 \%$ (range 1.2 - 34.0\%). Changes in left ventricular functional indices are shown in Table 2. Twenty seven (59\%) patients had evidence of MVO on the baseline LGE images, and in 16 (35\%) patients there was global LV remodeling. At follow-up 39 patients were NYHA class I, 4 patients class II, 2 patients class III and 1 patient class IV.

The mean surface-to-surface distance between manual and automatic segmentations was $1.2 \mathrm{~mm} \pm 0.3$ for the endocardium and $1.5 \mathrm{~mm} \pm 0.2$ for the epicardium.

\section{Local left ventricular remodeling}

Remodeling within infarcted myocardium was greater than in remote myocardium $(1.6 \% \pm 1.0$ vs $0.3 \% \pm 0.9, \mathrm{CI}$ : $-2.4 \%--0.2 \%, \mathrm{P}=0.02)$. One-way ANOVA revealed that infarct thickness had a significant effect on the degree of local remodeling at one year $(\mathrm{P}<0.0001)$ (Figure 5) with wall dilatation observed when infarct transmurality exceeded 50\%. Local remodeling was more severe when infarcts contained regions of $\mathrm{MVO}(3.8 \% \pm 1.3$ vs $-1.6 \% \pm 1.4, \mathrm{CI}:-9.1 \%--1.5 \%, \mathrm{P}=0.007)$ and when enddiastolic volume had increased by $>20 \%(4.8 \% \pm 1.4$ vs $-0.15 \% \pm 1.2, \mathrm{CI}:-8.9 \%--0.9 \%, \mathrm{P}=0.017)$.

\section{Wall thickness}

Mean wall thickness averaged throughout the acutely infarcted region decreased significantly during the first year $(10.1 \mathrm{~mm} \pm 0.3$ vs $8.9 \mathrm{~mm} \pm 1.7, \mathrm{CI}: 0.5-2.0$, $\mathrm{P}=0.002)$ and was unchanged in the remote region (7.1 $\mathrm{mm} \pm 0.2$ vs $7.1 \mathrm{~mm} \pm 0.2$, CI: $-0.5-0.5, \mathrm{P}=0.95)$. There was no difference in infarct wall thickness when either global left ventricular remodeling $(9.3 \mathrm{~mm} \pm 0.5 \mathrm{vs}$ $-8.7 \mathrm{~mm} \pm 0.3, \mathrm{CI}:-1.7-0.4, \mathrm{P}=0.24)$, or $\mathrm{MVO}$ was present $(9.0 \mathrm{~mm} \pm 0.3$ vs $-8.8 \mathrm{~mm} \pm 0.4, \mathrm{CI}:-1.2-0.9$, $\mathrm{P}=0.75)$.

\section{Discussion}

In this study we have used image-registration to map in three dimensions where the left ventricular wall dilates 

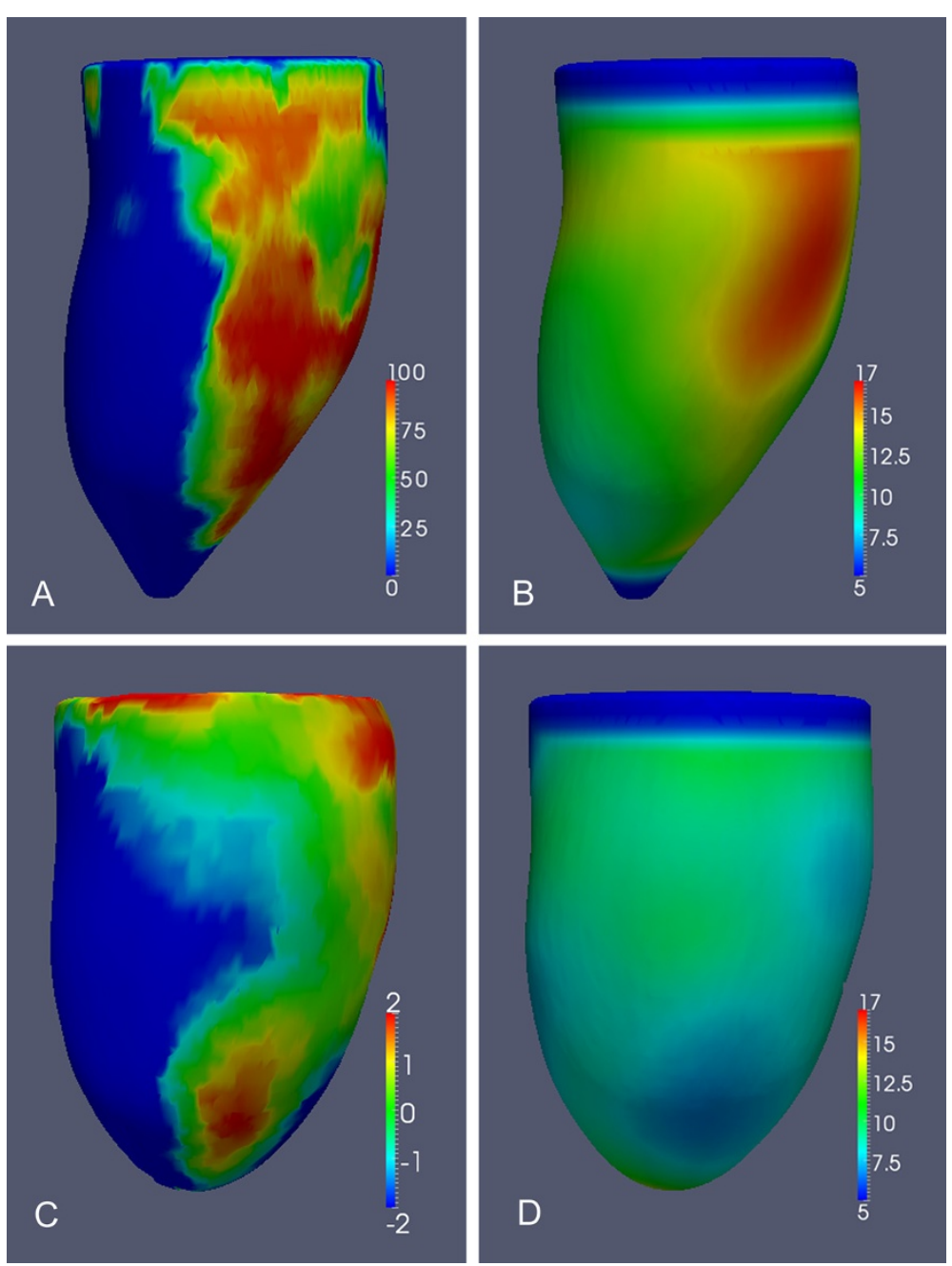

Figure 4 (A - D). 3D models of the left ventricle in an 51 year old male patient following primary percutaneous coronary intervention (PPCI) to a left circumflex artery occlusion. The images show co-registered data from segmented cine and Late Gadolinium Enhancement (LGE) images. (A) Distribution of enhancing necrosis (transmural percentage of infarcted myocardium) at baseline. (B) Left ventricular wall thickness $(\mathrm{mm})$ at baseline. (C) Local myocardial remodeling between baseline and follow-up (percentage expansion). (D) Left ventricular wall thickness at follow-up $(\mathrm{mm})$. These images demonstrate the anatomic relationship between the zone of infarction and the extent of local remodeling and wall thinning.

in the first year following STEMI and to relate this to the extent of local ischemic injury. Our findings show that following PPCI with standard medical therapy infarct transmurality and MVO have a significant effect on local ventricular wall remodeling while only modest dilatation is observed within non-ischemic myocardium.

Image co-registration is an emerging technique for the statistical analysis and quantification of cardiac motion and geometry [8]. In this study we used a labeled atlas to provide prior knowledge of cardiac anatomy to assist with image segmentation and as a template onto which different MR sequences are co-registered. This approach has been successfully used for determining shape variation of the left ventricle within patient populations $[18,19]$. It is also applicable to analyzing dynamic changes in cardiac morphology by using intrinsic signal intensity variations and anatomical features in the heart to track myocardial deformation over time - a process analogous to the explicit tags used in strain imaging $[20,21]$. In this work we used normalized mutual information between the myocardial segmentations at baseline and follow-up to map the chronic regional structural adaptations that occur within the left ventricle in response to STEMI. We also co-registered the anatomical images with the infarct segmentation enabling us to compare the severity of local remodeling with the extent of transmural necrosis at each point in the LV. This approach provides an appealing new methodology for assessing temporal changes in left ventricular morphology in relation to local ischemic injury. 
Table 1 Baseline characteristics of the patients. Stratified by which patients developed remodeling at follow-up

\begin{tabular}{|c|c|c|c|}
\hline & Remodeled & $\begin{array}{l}\text { Non- } \\
\text { remodeled }\end{array}$ & All \\
\hline Age (y) & $54 \pm 12$ & $53 \pm 10$ & $55 \pm 10$ \\
\hline \multicolumn{4}{|l|}{ Sex } \\
\hline Male & 16 & 28 & 44 \\
\hline Female & 0 & 2 & 2 \\
\hline Body Surface Area $\left(\mathrm{kg} / \mathrm{m}^{2}\right)$ & $1.91 \pm 0.33$ & $1.96 \pm 0.2$ & $1.97 \pm 0.18$ \\
\hline Systolic blood pressure (mmHg) & $131 \pm 32$ & $128 \pm 28$ & $133 \pm 28$ \\
\hline Diastolic blood pressure $(\mathrm{mmHg})$ & $83 \pm 22$ & $85 \pm 22$ & $84 \pm 20$ \\
\hline Heart Rate $\left(\mathrm{min}^{-1}\right)$ & $73 \pm 18$ & $73 \pm 13$ & $75 \pm 16$ \\
\hline Diabetes mellitus (\%) & $19 \%$ & $13 \%$ & $15 \%$ \\
\hline Current or ex-smoker (\%) & $44 \%$ & $67 \%$ & $59 \%$ \\
\hline Hypertension (\%) & $38 \%$ & $37 \%$ & $37 \%$ \\
\hline $\begin{array}{l}\text { Time from symptoms to } \\
\text { reperfusion (hours) }\end{array}$ & $3.5 \pm 2.6$ & $3.4 \pm 3.0$ & $3.5 \pm 2.6$ \\
\hline Peak Creatinine kinase (IU/L) & $2216 \pm 1994$ & $1775 \pm 1581$ & $2240 \pm 2034$ \\
\hline Peak Troponin I ( $\mu \mathrm{g} / \mathrm{L})$ & $65.0 \pm 78.7$ & $63.6 \pm 92.2$ & $64 \pm 80$ \\
\hline \multicolumn{4}{|l|}{ Culprit coronary artery (\%) } \\
\hline Left anterior descending & 56 & 53 & 54 \\
\hline Circumflex artery & 6 & 17 & 13 \\
\hline Right coronary & 38 & 30 & 33 \\
\hline \multicolumn{4}{|l|}{ TIMI flow Pre-PPCI (\%) } \\
\hline Grade 0 & 94 & 80 & 85 \\
\hline Grade I & 6 & 3 & 9 \\
\hline Grade II & 0 & 7 & 4 \\
\hline Grade III & 0 & 0 & 0 \\
\hline \multicolumn{4}{|l|}{ TIMI flow Post-PPCI (\%) } \\
\hline Grade II & 6 & 0 & 4 \\
\hline Grade III & 63 & 100 & 96 \\
\hline \multicolumn{4}{|l|}{ Discharge medication (\%) } \\
\hline Aspirin & 100 & 100 & 100 \\
\hline Clopidogrel & 96 & 96 & 96 \\
\hline Beta blocker & 94 & 90 & 92 \\
\hline $\mathrm{Ca}^{2+}$ channel blocker & 6 & 0 & 2 \\
\hline $\begin{array}{l}\text { Angiotensin converting } \\
\text { enzyme inhibitor }\end{array}$ & 98 & 94 & 96 \\
\hline Statin & 98 & 98 & 98 \\
\hline
\end{tabular}

Values are means \pm standard deviations. $P P C l=$ Primary percutaneous coronary intervention. $L V=$ Left Ventricle.

A limitation of conventional imaging is that assessment of cavity volumes does not reflect the dynamic regional changes in myocardial structure that occur in response to ischemia-reperfusion injury. In animal studies of remodeling there is an acute phase of lateral slippage of the myocardial planes within the infarct leading to progressive left ventricular dilatation [22]. In the later
Table 2 Comparison of left ventricular functional indices obtained at baseline and 1 year follow-up

\begin{tabular}{lccc}
\hline & Baseline & Follow-up & P value \\
\hline LV mass (g) & $155 \pm 44$ & $133 \pm 33$ & $<0.001$ \\
LV end diastolic volume $(\mathrm{ml})$ & $146 \pm 38$ & $160 \pm 46$ & 0.004 \\
LV end systolic volume $(\mathrm{ml})$ & $67 \pm 26$ & $71 \pm 31$ & 0.4 \\
Stroke Volume $(\mathrm{ml})$ & $80 \pm 19$ & $90 \pm 28$ & 0.006 \\
Ejection Fraction $(\%)$ & $56 \pm 9$ & $57 \pm 12$ & 0.18 \\
\hline
\end{tabular}

Values are mean \pm standard deviations. $L V=$ Left Ventricle.

stages of disease non-ischemic myocardium may develop a maladaptive response of ventricular dilatation which ultimately leads to heart failure [5,23]. Left ventricular remodeling is also determined by factors other than initial infarct size $[17,24]$ and both infarct transmurality and the presence of persistent MVO influence the development of chronic heart failure [25-27]. Despite revascularization and medical therapy $35 \%$ of the patients in our study developed global remodeling as defined by end diastolic volumes. Our findings indicate that infarcted myocardium plays a much greater role in such chronic remodeling than non-ischemic tissue. Our observation of progressive dilatation in the ischemic territory is consistent with elongation of the infarcted myocardium which typically develops during early post-infarction remodeling [28]. The extent of transmural necrosis is known to influence global remodeling [27] and we observed that local myocardial dilatation occurs when infarct thickness exceeds 50\%. This is consistent with findings that acute sub-endocardial infarctions remain viable with the potential for contractile recovery [29]. We also observed the highest rates of local wall dilatation when infarcts had regions of MVO, which is recognized as a predictor of adverse remodeling [16]. We observed a significant decrease in left ventricular mass and wall thickness in the infarcted region over time and this is consistent with transient ischemia-reperfusion edema within the infarct zone [30].

In our cohort remodeling in the non-ischemic territory was modest compared to the infarcted region. The late phase of ventricular remodeling has been shown to be amendable to medical therapy, as chronic administration of angiotensin-converting enzyme inhibitors (ACEIs) are associated with a reduction in the extent of ventricular dilation $[4,31]$ and prolonged therapy with $\beta$-blockers may limit or even reverse remodeling and dysfunction $[32,33]$. The great majority of patients in this study were prescribed both ACEIs and $\beta$-blockers and our findings suggest that this therapy may be effective in limiting the development of maladaptive remodeling within remote myocardium following successful PPCI.

Image registration offers a more refined model of left ventricular adaptations to infarction than can be derived 


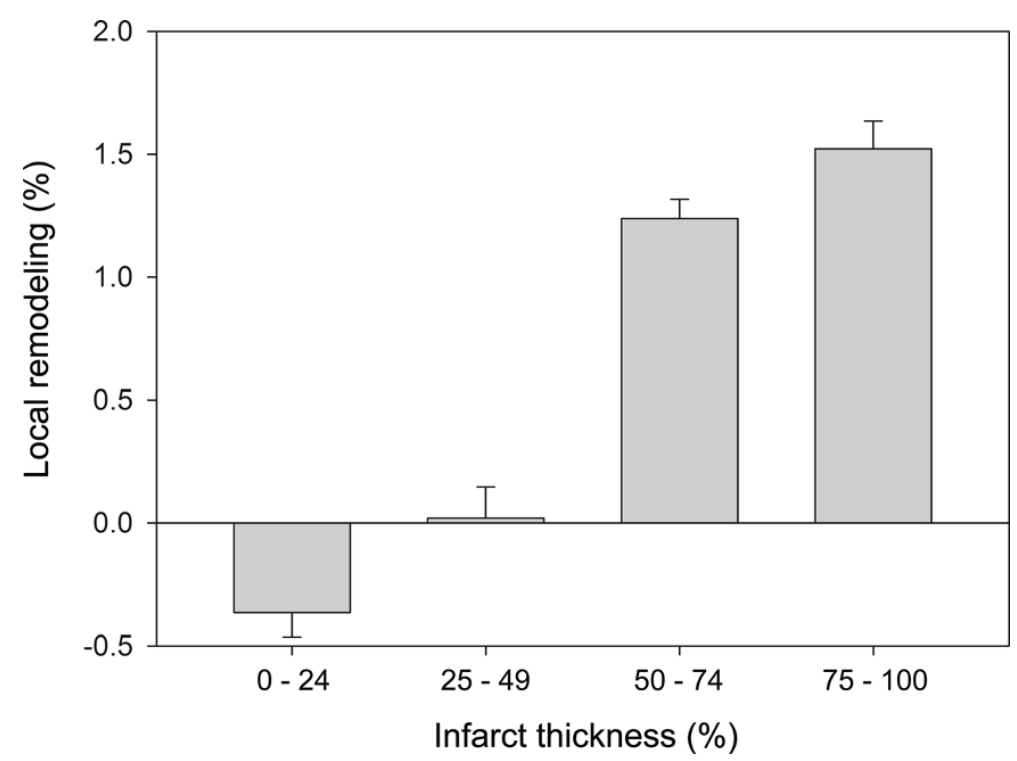

Figure 5 The relationship between local remodeling and infarct transmurality (Mean values with $95 \%$ confidence interval). The transmural extent of infarction at baseline has a significant influence on the severity myocardial remodeling at the same location in the left ventricle (ANOVA P $<0.0001$ ). Local ventricular expansion is most marked when $\geq 50 \%$ of the wall is infarcted.

from global measures of cavity volume. The potential clinical applications include interventional studies of cardio-protective therapies where the physiological effects of remodeling within infarcted and remote myocardium may be separately measured and compared to imaging markers of ischemic injury. The approach may also be helpful for predicting the complications of ischemic remodeling such as ventricular aneurysm formation and the development of ischemic mitral regurgitation. Developments in parallel imaging also make 3D acquisition of both cine images and LGE appealing for registration techniques as they avoid section mis-registration [34-36].

We do not have inter-study reproducibility data for the registration technique as the patients were only scanned once at each visit. We used the segmentation of the myocardium to guide registration so the final transformation is weighted to geometric changes within the myocardium. We also used an initial non-rigid transformation to limit the influence of local structures other than myocardium. However, as the mutual information between images is not explicitly defined the registration may also be influenced by structures immediately adjacent to the heart. EM segmentation provided an accurate segmentation of the myocardial boundaries, but the influence of signal intensity differences in acute and remote myocardium was not evaluated. Mean wall thickness in infarcted myocardium at follow-up was $8.9 \mathrm{~mm}$ and so there were typically at least 4 voxels across the width of the LV to perform the non-rigid registration However, accuracy of registration may be limited by severely thinned myocardium. We did not use follow-up LGE images in the remodeling analysis as we wanted to distinguish expansion of remodeled myocardium from expansion of the zone of necrosis. We also did not segment the $\mathrm{T} 2$ images due to the relatively low signal to noise ratio of these sequences.

In our cohort the mean baseline LVEF was not significantly impaired and this is likely to have been influenced by the exclusion of subjects with acutely impaired left ventricular function who were not suitable for CMR within 7 days of PPCI. However, the observation that local remodeling may be observed despite a normal ejection fraction supports the premise that global indices of function may mask the regional effects of ischemic damage. Further research is needed to explore whether local morphological changes affect long-term outcome or are independent predictors of heart failure. GMM has not been formally assessed for infarct segmentation although the methodology is similar to validated techniques for pixel-weighted infarct quantification [37]. Our findings are limited to assessing structural remodeling and we did not measure regional strain or wall thickening to evaluate changes in contractile function as a consequence of STEMI [38]. Further work is also needed to establish the correspondence between image-based transformations and ultra-structural adaptations within the myocardium.

\section{Conclusions}

The severity of ischemic injury has a significant effect on local ventricular wall remodeling while only modest 
dilatation is observed within non-ischemic myocardium. This indicates that revascularization and standard medical therapy may be effective at preventing structural changes in the remote myocardium, and that further limitation of chronic remodeling may depend on therapies directed at modulating ischemia-reperfusion injury.

Co-registration of CMR images acquired in longitudinal studies is an analysis technique which has potential for assessing dynamic changes in ventricular structure in relation to therapeutic interventions.

\begin{abstract}
Abbreviations
AAR: Area-at-risk; ACEl: Angiotensin-converting enzyme inhibitor; CMR: Cardiac magnetic resonance; LGE: Late gadolinium enhancement; MVO: Microvascular obstruction; NYHA: New York Heart Association Functional Classification; PPCl: Primary percutaneous coronary intervention; STEMI: ST-segment elevation myocardial infarction; T2-SPIR: T2 weighted spectrally selective inversion recovery; TIMI: Thrombolysis in Myocardial Infarction trial grading.
\end{abstract}

\section{Competing interests}

The authors declare that they have no competing interests.

\section{Acknowledgments}

We wish to thank our research nurses Clare Neuwirth and Yvonne Tan. The study was funded by the Medical Research Council and a British Heart Foundation Research Excellence Award.

\section{Author details}

${ }^{1}$ Robert Steiner MRI Unit, MRC Clinical Sciences Centre, Imperial College London, Hammersmith Hospital Campus, Du Cane Road, London W12 ONN, UK. ${ }^{2}$ Department of Computing, Imperial College London, South Kensington Campus, Exhibition Road, London SW7 2AZ, UK. ${ }^{3}$ Department of Imaging, Imperial College Healthcare NHS Trust, Hammersmith Hospital, Du Cane Road, London W12 OHS, UK. ${ }^{4}$ Department of Cardiology, Imperial College Healthcare NHS Trust, London, Hammersmith Hospital, Du Cane Road, London W12 OHS, UK.

\section{Authors' contributions}

DPO'R conceived of the study design, performed the statistical analysis and drafted the manuscript. WS developed the co-registration methods and analyzed the data. BA participated in the coordination of the study and revising the manuscript. AJB contributed to the manual data analysis and revising the manuscript. GD scanned the patients, contributed to data analysis and revised the manuscript. DR participated in the study design and in developing the image analysis methods. SAC conceived of the research program, coordinated the study and revised the manuscript. All authors read and approved the final manuscript.

Received: 10 January 2012 Accepted: 21 June 2012

Published: 21 June 2012

\section{References}

1. Keeley EC, Boura JA, Grines CL. Primary angioplasty versus intravenous thrombolytic therapy for acute myocardial infarction: a quantitative review of 23 randomised trials. The Lancet. 2003;361:13-20.

2. Gaudron P, Eilles C, Kugler I, Ertl G. Progressive left ventricular dysfunction and remodeling after myocardial infarction. Potential mechanisms and early predictors. Circulation. 1993;87:755-763.

3. Weisman HF, Bush DE, Mannisi JA, Weisfeldt ML, Healy B. Cellular mechanisms of myocardial infarct expansion. Circulation. 1988;78:186-201.

4. Mitchell GF, Lamas GA, Vaughan DE, Pfeffer MA. Left ventricular remodeling in the year after first anterior myocardial infarction: a quantitative analysis of contractile segment lengths and ventricular shape. J Am Coll Cardiol. 1992:19:1136-1144.

5. Braunwald E, Pfeffer MA. Ventricular enlargement and remodeling following acute myocardial infarction: mechanisms and management. Am J Cardiol. 1991;68:6.
6. Landmesser U, Wollert KC, Drexler H. Potential novel pharmacological therapies for myocardial remodelling. Cardiovasc Res. 2009;81:519-527.

7. Mazziotta JC, Toga AW, Evans A, Fox P, Lancaster J. A Probabilistic Atlas of the Human Brain: Theory and Rationale for Its Development: The International Consortium for Brain Mapping (ICBM). Neuroimage. 1995;2:89-101.

8. Fonseca CG, Backhaus M, Bluemke DA, Britten RD, Chung JD, Cowan BR, Dinov ID, Finn JP, Hunter PJ, Kadish AH, et al. The Cardiac Atlas Project-an imaging database for computational modeling and statistical atlases of the heart. Bioinformatics. 2011;27:2288-2295.

9. TIMI Study Group. The Thrombolysis in Myocardial Infarction (TIMI) trial. Phase I findings. N Engl J Med. 1985;312:932-936.

10. Lorenzo-Valdes M, Sanchez-Ortiz Gl, Elkington AG, Mohiaddin RH, Rueckert D. Segmentation of $4 D$ cardiac MR images using a probabilistic atlas and the EM algorithm. Med Image Anal. 2004:8:255-265.

11. Zhuang X, Rhode KS, Razavi RS, Hawkes DJ, Ourselin S. A registration-based propagation framework for automatic whole heart segmentation of cardiac MRI. IEEE Trans Med Imaging. 2010;29:1612-1625.

12. Valindria W, Angue M, Vignon N, Walker PM, Cochet A, Lalande A. Automatic Quantification of Myocardial Infarction from Delayed Enhancement MRI. Dijon, France: In 2011 Seventh International Conference on Signal-Image Technology and Internet-Based Systems (SITIS); 2011. p. 277-283.

13. Grevera GJ, Udupa JK. Shape-based interpolation of multidimensional grey-level images. IEEE Trans Med Imaging. 1996;15:881-892.

14. Lorensen WE, Cline HE. Marching cubes: A high resolution 3D surface construction algorithm. [Abstract]. Computer graphics. 1987;21:163-169.

15. Pfeffer MA, Braunwald E. Ventricular remodeling after myocardial infarction. Experimental observations and clinical implications. Circulation. 1990:81:1161-1172.

16. Bogaert J, Kalantzi M, Rademakers FE, Dymarkowski S, Janssens S. Determinants and impact of microvascular obstruction in successfully reperfused ST-segment elevation myocardial infarction. Assessment by magnetic resonance imaging. Eur Radiol. 2007;17:2572-2580.

17. Bolognese L, Neskovic AN, Parodi G, Cerisano G, Buonamici P, Santoro GM, Antoniucci D. Left ventricular remodeling after primary coronary angioplasty: patterns of left ventricular dilation and long-term prognostic implications. Circulation. 2002;106:2351-2357.

18. Ardekani S, Weiss RG, Lardo AC, George RT, Lima JAC, Wu KC, Miller MI, Winslow RL, Younes L. Computational method for identifying and quantifying shape features of human left ventricular remodeling. Ann Biomed Eng. 2009;37:1043-1054.

19. Young AA, Frangi AF. Computational cardiac atlases: from patient to population and back. Exp Physiol. 2009;94:578-96.

20. Chandrashekara R, Mohiaddin RH, Rueckert D. Analysis of 3-D myocardial motion in tagged MR images using nonrigid image registration. Medical Imaging, IEEE Transactions on. 2004:23:1245-1250.

21. Hor KN, Gottliebson WM, Carson C, Wash E, Cnota J, Fleck R, Wansapura J, Klimeczek P, Al-Khalidi HR, Chung ES, et al. Comparison of Magnetic Resonance Feature Tracking for Strain Calculation With Harmonic Phase Imaging Analysis. J Am Coll Cardiol Img. 2010;3:144-151.

22. Olivetti G, Capasso JM, Sonnenblick EH, Anversa P. Side-to-side slippage of myocytes participates in ventricular wall remodeling acutely after myocardial infarction in rats. Circ Res. 1990;67:23-34.

23. Stillwell GK. The Law of Laplace. Some clinical applications. Mayo Clin Proc. 1973:48:863-869.

24. De Luca L, Sardella G, Davidson CJ, De Persio G, Beraldi M, Tommasone T, Mancone M, Nguyen BL, Agati L, Gheorghiade M, Fedele F. Impact of intracoronary aspiration thrombectomy during primary angioplasty on left ventricular remodelling in patients with anterior ST elevation myocardial infarction. Heart. 2006;92:951-957.

25. Morishima I, Sone T, Okumura K, Tsuboi H, Kondo J, Mukawa H, Matsui H, Toki Y, Ito T, Hayakawa T. Angiographic no-reflow phenomenon as a predictor of adverse long-term outcome in patients treated with percutaneous transluminal coronary angioplasty for first acute myocardial infarction. J Am Coll Cardiol. 2000;36:1202-1209.

26. Ito H, Maruyama A, Iwakura K, Takiuchi S, Masuyama T, Hori M, Higashino Y, Fujii K, Minamino T. Clinical Implications of the 'No Reflow' Phenomenon: A Predictor of Complications and Left Ventricular Remodeling in Reperfused Anterior Wall Myocardial Infarction. Circulation. 1996;93:223-228.

27. Tarantini G, Razzolini R, Cacciavillani L, Bilato C, Sarais C, Corbetti F, Marra MP, Napodano M, Ramondo A, lliceto S. Influence of transmurality, infarct 
size, and severe microvascular obstruction on left ventricular remodeling and function after primary coronary angioplasty. Am J Cardiol. 2006;98:1033-1040.

28. Konstam MA, Kramer DG, Patel AR, Maron MS, Udelson JE. Left ventricular remodeling in heart failure: current concepts in clinical significance and assessment. JACC Cardiovasc Imaging. 2011:4:98-9108.

29. Choi KM, Kim RJ, Gubernikoff G, Vargas JD, Parker M, Judd RM. Transmural extent of acute myocardial infarction predicts long-term improvement in contractile function. Circulation. 2001;104:1101-1107.

30. Friedrich MG. Tissue Characterization of Acute Myocardial Infarction and Myocarditis by Cardiac Magnetic Resonance. J Am Coll Cardiol Img. 2008; 1:652-662.

31. Pfeffer MA, Braunwald E. Ventricular enlargement following infarction is a modifiable process. Am J Cardiol. 1991;68:127D-131D.

32. Doughty RN, Whalley GA, Walsh HA, Gamble GD, Lopez-Sendon J, Sharpe N. Effects of Carvedilol on Left Ventricular Remodeling After Acute Myocardial Infarction. Circulation. 2004;109:201-206.

33. Hall S, Cigarroa C, Marcoux L, Risser R, Grayburn P, Eichhorn E. Time course of improvement in left ventricular function, mass and geometry in patients with congestive heart failure treated with beta-adrenergic blockade. J Am Coll Cardiol. 1995;25:1154-1161.

34. Parish V, Hussain T, Beerbaum P, Greil G, Nagel E, Razavi R, Schaeffter T, Uribe $S$. Single breath-hold assessment of ventricular volumes using 32-channel coil technology and an extracellular contrast agent. J Magn Reson Imaging. 2010;31:838-844.

35. Davarpanah AH, Chen Y-P, Kino A, Farrelly CT, Keeling AN, Sheehan JJ, Ragin AB, Weale PJ, Zuehlsdorff S, Carr JC. Accelerated Two- and Threedimensional Cine MR Imaging of the Heart by Using a 32-Channel Coil1. Radiology. 2010;254:98-108.

36. Peters DC, Appelbaum EA, Nezafat R, Dokhan B, Han Y, Kissinger KV, Goddu $B$, Manning WJ. Left ventricular infarct size, peri-infarct zone, and papillary scar measurements: A comparison of high-resolution $3 \mathrm{D}$ and conventional 2D late gadolinium enhancement cardiac MR. J Magn Reson Imaging. 2009;30:794-800.

37. Heiberg E, Ugander M, Engblom H, Gotberg M, Olivecrona GK, Erlinge D, Arheden $\mathrm{H}$. Automated quantification of myocardial infarction from MR images by accounting for partial volume effects: animal, phantom, and human study. Radiology. 2008;246:581-588.

38. Bogaert J, Bosmans H, Maes A, Suetens P, Marchal G, Rademakers FE. Remote myocardial dysfunction after acute anterior myocardial infarction: impact of left ventricular shape on regional function: A magnetic resonance myocardial tagging study. J Am Coll Cardiol. 2000;35:1525-1534.

\section{doi:10.1186/1532-429X-14-41}

Cite this article as: O'Regan et al:: Remodeling after acute myocardial infarction: mapping ventricular dilatation using three dimensional CMR image registration. Journal of Cardiovascular Magnetic Resonance 2012 14:41.

\section{Submit your next manuscript to BioMed Central and take full advantage of:}

- Convenient online submission

- Thorough peer review

- No space constraints or color figure charges

- Immediate publication on acceptance

- Inclusion in PubMed, CAS, Scopus and Google Scholar

- Research which is freely available for redistribution 